\title{
Walter Dorwing Teague: precursor de la identidad corporativa
}

\section{WalterDorwin Teague: pioneer of the corporate identity}

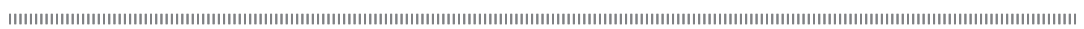

(D) Francisco Silvestre Navarro

Escuela de Arquitectura y Politécnica, Universidad Europea de Valencia, España

fsilvestre@fransilvestrearquitectos.com

Pablo Camarasa Balaguer

Escuela de Arquitectura y Politécnica, Universidad Europea de Valencia, España camarasa.b.pablo@gmail.com

\section{Resumen}

La consolidación de la figura del diseñador industrial en Estados Unidos durante las décadas centrales del siglo XX está íntimamente ligada a la aparición de grandes marcas y a la identidad de éstas. Los diseñadores americanos se especializaron dando forma a una nueva profesión, pusieron en marcha una inteligencia compartida y multidisciplinar y, quizás sin saberlo, fueron pioneros en desdibujar los límites entre las disciplinas creativas y en convertirlas en una parte importante de las empresas. Se trata de un valioso modelo de transformación y adaptabilidad del que se pueden extraer conclusiones y paralelismos con la época actual. El presente texto se centra en la figura del diseñador Walter Dorwin Teague, quien guiado por la intuición de la belleza influyó en la industria estadounidense a partir de la década de 1920, dotándola de una identidad necesaria para su perpetuación a lo largo del tiempo. Se exponen algunos de sus trabajos más influyentes con la finalidad de poder valorar el impacto que este profesional causó mientras estuvo activo y tomar conciencia del legado tan rico que dejó para la posteridad.

Palabras clave: Diseño industrial, Arquitectura, Identidad, Empresa, Estados Unidos.

\author{
Artículo original / Original Article \\ Correspondencia / Correspondence \\ fsilvestre@fransilvestrearquitectos.com \\ camarasa.b.pablo@gmail.com \\ Financiación / Fundings \\ Sin financiación \\ Recibido / Recived: 19/04/2021 \\ Aceptado / Accepted: 3/09/2021 \\ Publicado / Published: 17/12/2021 \\ Cómo citar este trabajo. \\ How to cite this paper. \\ Silvestre Navarro, F., Camarasa \\ Balaguer, P. (2021). Walter \\ Dorwin Teague: Precursor de la \\ identidad corporativa. i+Diseño. \\ Revista Internacioan de \\ Innovación, Investigación y \\ Desarrollo en Diseño, 16. \\ DOI: 10.24310/ \\ Idiseno.2021.v16i.12386
}




\section{Abstract}

The consolidation of the figure of the industrial designer in the United States during the central decades of the 20th century is closely linked to the appearance of great brands and their identity. American designers specialized and shaped a new profession, they launched a shared and multidisciplinary intelligence and they were pioneers in blurring the boundaries between creative disciplines and in making them an important part of companies. It is a valuable model of transformation and adaptability from which conclusions and parallelisms can be drawn with the current era. This article focuses on the figure of the designer Walter Dorwin Teague, who guided by the intuition of beauty influenced American industry from the 1920s, giving it a necessary identity for its perpetuation over time. Some of his most influential works are exposed in this text in order to assess the impact that this professional caused while he was active and to become aware of the rich legacy left for posterity.

Keywords: Industrial design, Architecture, Identity, Company, United States.

Los orígenes del diseño industrial norteamericano estuvieron representados por creativos como Raymond Loewy (Porter, 2002), Norman Bel Geddes, Henry Dreyfuss y el propio Walter Dorwin Teague, que da pie a este artículo, entre otros ${ }^{1}$. Es cierto que en numerosas publicaciones se habla de la superficialidad de la corriente styling con la que todos ellos se identificaban, así como de su escasa carga teórica. Pero ya desde un punto de vista estético, una vertiente más contenida en la que se trató de aunar el diseño de formas elegantes y atractivas y la puesta en práctica de una aproximación científica a estas creaciones, fue la representada por el citado Teague. Sus obras estaban basadas en un método científico y racional capaz de garantizar un producto bello y técnicamente bien resuelto. De ese modo, podían legitimar, de cara a las empresas que contrataban sus servicios, la validez y efectividad de la profesión de diseñador industrial apelando al carácter metódico de la misma, a la vez que justificar la creación de un producto atractivo y práctico para el consumidor (Lippincott, 1945) que pudiese actuar como elemento identificador de la compañía.

\section{Un pionero procedente del mundo de la publicidad}

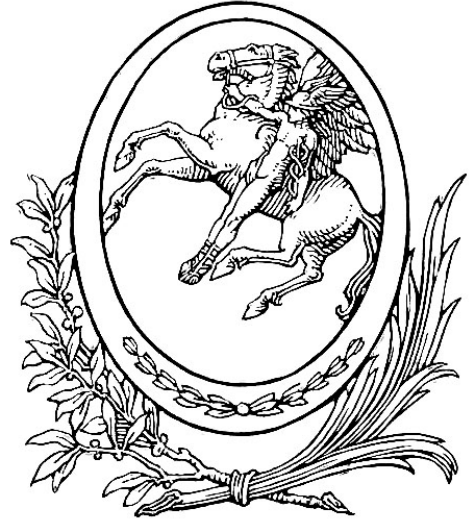

Figura 1.

Logotipo de Pynson Printers diseñado por Walter Dorwin Teague. Fuente: Archivo propio.
Walter Dorwin Teague nació en el año 1883 y se inició en el mundo laboral trabajando para el periódico de la localidad en la que residía como reportero. En 1903 se desplazó hasta Nueva York, donde se formó en pintura en la Art Students League of New York. Ya finalizada su etapa de formación académica, trabajó en primer término como representante comercial para algunas revistas, entre las que destacó su vinculación con Time Magazine.

Posteriormente ejerció como tipógrafo, campo creativo en el que llegaría a convertirse en una autoridad, llevando a cabo diseños que pasaron a ser conocidos como Teague Borders (Soloway \& London, 2007) y que llegarían a ser estandarizados y empleados por otros diseñadores. Trabajó también como ilustrador, con unos resultados tan positivos que llevaron al empresario Walter Whitehead a ofrecerle un contrato de trabajo para la agencia de publicidad Ben Hampton, en la que empezaría a desarrollar anuncios (Hornung, 1991) y a conocer estrategias de comunicación. El propio Whitehead

1. A este respecto, véase Gantz, C. (2014). Founders of American Industrial Design. Jefferson, NC: McFarland and Company Inc. 
le ayudaría a abrirse camino en sus inicios, y cuando éste dejó su puesto en la compañía para ocupar un cargo mejor en la destacada Calkins \& Holden el año 1908, le propuso a Teague que se marchara con él, lo cual le trasladaba hasta un buen lugar para observar la dinámica contemporánea y las necesidades del mercado estadounidense.

Su relación laboral con la empresa de Earnest Elmo Calkins se prolongó hasta 1912, año en el que se desvinculó de la misma para abrir su propio estudio, centrado en el desarrollo tipográfico, aunque también realizaba trabajos de interiorismo. Su estilo fue pronto ampliamente reconocido desde los primeros años de trabajo. En la década de los años veinte extendió su campo de actuación al diseño gráfico y el diseño de producto. Fundó, junto a Elmer Adler, Hubert Canfield y David Silve la empresa Pynson Printers, de la que el propio Teague se encargaría de elaborar el logotipo.

Su curiosidad le llevó a viajar en 1926 al viejo continente, donde conoció nuevos diseños y materiales. Allí descubrió el trabajo de Le Corbusier, y una vez regresó a Nueva York, decidió centrar su trabajo en el diseño o re-estilismo de productos. Fue en el año 1927 cuando inició su andadura como diseñador industrial, siendo considerado por la sociedad como uno de los pioneros en este campo y a convertirse en uno de los profesionales norteamericanos más prolíficos de su tiempo. Contaba con 43 años cuando estableció una nueva empresa centrada en el desarrollo y diseño de productos, incluyendo en su tarjeta de contacto y en el nombre de su negocio las palabras "diseño industrial». En ese mismo año, y junto a otros diseñadores como Raymond Loewy, Norman Bel Geddes o Henry Dreyfuss, constituyeron la American Union of Decorative Artists and Craftsmen, comenzando a establecer, desde un punto de vista comercial, las bases de aquella nueva profesión.

Su primer cliente fue Eastman Kodak, que se mantendría durante los siguientes treinta años en su cartera. En aquellos tiempos, dicha compañía estaba considerando la posibilidad de incorporar a su plantilla la figura de un artista con la finalidad de que reinterpretase estéticamente sus productos. El conservador del Museo Metropolitano de Arte de Nueva York, Richard Bach, recomendó a Teague al directivo de Eastman Kodak Adolph Stuber (Feinberg, 1972). A partir de aquellos momentos se inició una colaboración profesional que daría como resultado cámaras fotográficas muy conocidas entre las que se incluyen la Vanity Kodak (Hall \& Hall, 2006), aparecida en 1928, la Deco Gift Camera también en ese mismo año, planteada en principio como regalo de navidad; la Baby Brownie producida a partir de 1934, la Bantam Special (Gautrand, 1983), uno de sus modelos más reconocidos, fabricada entre 1936 y 1948, y la Brownie Hawkeye, que apareció en 1950 (Soloway \& London, 2006). Destacó su diseño para la primera cámara tipo Polaroid para Edwin Land en 1948. La relación entre Teague y Kodak fue tan prolífica y beneficiosa para ambas partes que en 1934, la compañía de máquinas fotográficas creó una división centrada en la estética de sus productos en la que nombró a Teague su asesor, pasando a ejercer a modo de director creativo e influyendo sobre la identidad de la marca.

\section{Una metodología: Styling, Streamline y la identidad corporativa}

La popularidad de Teague vino impulsada por los trabajos que realizó para Kodak, lo que le valió que desde 1929 a 1931 el número de clientes se multiplicase. Desarrolló planes de estrategia para diferentes empresas que producían objetos dirigidos a todo tipo de público, y fue pionero en su profesión diseñando el año 1933 el modelo 16

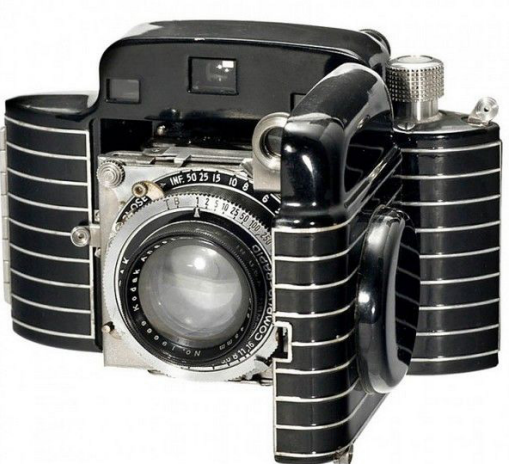

Figura 2.

Cámara fotográfica Kodak Bantam Special. Fuente: pinterest.com (Consultado el 19 de abril de 2021) 


\author{
Estableció una metodología \\ de trabajo y conceptual \\ que aplicó en las sucesivas \\ compañías que recurrieron \\ a sus servicios, obteniendo, \\ él como creativo, los \\ resultados deseados y \\ esperados, y ellas como \\ clientes, pingües beneficios.
}

para la empresa automovilística Marmon, siendo el primer coche concebido por un diseñador industrial.

Estableció una metodología de trabajo y conceptual que aplicó en las sucesivas compañías que recurrieron a sus servicios, obteniendo, él como creativo, los resultados deseados y esperados, y ellas como clientes, pingües beneficios.

Por un lado, el método conceptual comenzó basándose en el ya citado styling, que podría tenerse en cuenta como uno de los motores generadores de la presente investigación debido a que era el resultado, el ejemplo palpable, de aquel cambio que se sobrepuso a la crisis económica para salir adelante.

El término styling adquirió un significado de capital importancia en las décadas centrales del siglo XX, siendo considerado como una estrategia del diseño y venta de productos elaborados por la industria orientada a lograr el éxito comercial de las compañías. El concepto styling tenía como objetivo incrementar el numero de ventas y dinamizar el mercado para así reactivar el consumo recurriendo al atractivo de sus productos (Rodríguez Ortega, 2002) en un tiempo en el que la superación de la crisis del 29 era el primer y principal objetivo de la población estadounidense. Suponía la continuación de lo idéntico bajo ropajes distintos, considerando la actividad del diseñador como un medio para incrementar la facturación de las empresas, quedando subordinada a los intereses del productor. Esta técnica de mercado se introdujo por vez primera en la industria del automóvil, extendiéndose con presteza a otros ámbitos.

Teague entendió perfectamente el calado que este fenómeno podía llegar a tener en la sociedad norteamericana, y por ello se valió del mismo para numerosos trabajos. No obstante, fue un poco más allá adentrándose en el Streamline, un estilo que se apoyaba en el aerodinamismo, con formas que, además de funcionar muy bien entre el público, suponían menores costes de producción debido a que sus geometrías permitían una mayor inercia en la fabricación, utilizando menos material. Además, se podían fabricar productos con un menor número de piezas, lo cual economizaba su ensamblaje. La facilidad de fabricación permitía que los tiempos de producción fuesen también menores, alzándose como otra ventaja más.

Por otra parte, la capacidad de trabajo multidisciplinar que demostraba el estudio de Teague dio como resultado la aplicación del concepto de «identidad corporativa» que décadas atrás el alemán Peter Behrens había puesto en práctica con AEG, pues se llevaba a cabo una labor en la que se entrelazaban diferentes profesionales, expertos cada uno de ellos en un campo de trabajo distinto, aportando sus conocimientos para los objetivos técnicos y comerciales de la empresa. La idea de «identidad corporativa» empresarial se extendió por todo el territorio norteamericano durante las décadas de los años treinta y cuarenta a través de diferentes ferias y exposiciones en las que se mostraban las colaboraciones que ya empezaban a darse entre diferentes compañías e industrias, y el papel jugado por Teague para su desarrollo fue crucial.

Buscando transmitir sus ideas, y al igual que hicieron otros diseñadores de la época como el propio Loewy, escribió un libro que sería publicado bajo el título Design this day. The technique of order in the machine age (Teague, 1940), obteniendo una importante repercusión mediática y una gran aceptación entre el público especializado, y siendo considerado el primer libro cuya temática principal era la del diseño industrial. En él exploraba la dependencia que ya entonces tenían los ciudadanos de 
las máquinas y de la industria y analizaba cuál era el papel del diseñador dentro de la sociedad. Otros libros publicados por Teague fueron Land of plenty: A summary of possibilities, en colaboración con John Storck y publicado en 1947 (Teague, 1947), y Flour for man's bread. A history of milling, aparecido en $1952^{2}$. También escribió textos para entidades museísticas como fue el caso de aportaciones a los fondos del MoMA y artículos para numerosas publicaciones como las revistas Forbes, New Yorker, Art and Decoration, Business Week o Popular Mechanics. En esta última publicó un escrito titulado «Planning the world of tomorrow», aparecido en 1940 y en el que predecía un futuro mejor con la llegada de nuevos materiales industriales, ideas innovadoras y creativos diseños.

\section{Encargos y crecimiento profesional}

Durante la década de los cuarenta Walter Dorwin Teague empezó a colaborar con la compañía Boeing, estableciendo una oficina en Seattle bajo el nombre de Teague's Aviation Studios. Ésta se encargaría durante los siguientes sesenta años del diseño de los interiores de aeroplanos como el Boeing modelo 377 Stratocruiser o el modelo 747, además del nuevo Air Force Academy. En 1959, la cartera de clientes de Teague era muy variada, realizando trabajos para compañías como la cervecera Schaefer, la compañía de mensajería UPS, la General Foods Corporation, Steinway o para la marina de los Estados Unidos. Su popularidad rivalizaba incluso con la de Raymond Loewy, siendo ambos los dos profesionales dedicados al diseño industrial mejor considerados por los medios de comunicación.

Su implicación e influencia iba más allá. Así, en 1944 se convirtió en el primer presidente de la Sociedad de Diseñadores Industriales (SID) ${ }^{3}$ como consecuencia de sus logros y aportaciones para la formalización y consolidación de dicha organización, conformada en origen por Raymond Loewy, Henry Dreyfuss y otros quince destacados diseñadores de las costa este norteamericana.

Su producción arquitectónica fue también notable. Buscando crear una clasificación de la misma, se podrían establecer tres grupos: el de las gasolineras Texaco, el de los pabellones diseñados por la Ford Motor Company, y el de los edificios proyectados para la Feria de Nueva York del año 1939 (Cotter, 2009).

Empezando por el primer grupo, a finales de los años 20 la empresa Texaco, conocida antes como The Texas Company, que era ya en el siglo XX uno de los gigantes de la industria del combustible, compró otras dos compañías distribuidoras de petróleo, en un afán por expandirse por todos los estados del país y por introducir nuevos productos. A raíz de su establecimiento nacional a mediados de los años 30, la corporación inició una nueva campaña de marketing cuyos objetivos fundamentales consistían en modernizar el logotipo y establecer nuevas estaciones de servicio que contribuyesen a crear una imagen fácilmente identificable por la sociedad. Buscaban desarrollar una identidad corporativa.

2. A este respecto, véase Marquette, C. L. (1952) Flour for man's bread: a history of milling by John Storck and Walter Dorwin Teague. Minneapolis: University of Minnesota Press.

3. Hoy día, y desde que cambiara su nombre en 1965, la organización es conocida como IDSA (Industrial Designers Society of America). 
Figura 3.

Estación de servicio Texaco diseñada por Teague. Fuente: teague.com (Consultado el 26 de marzo de 2021).

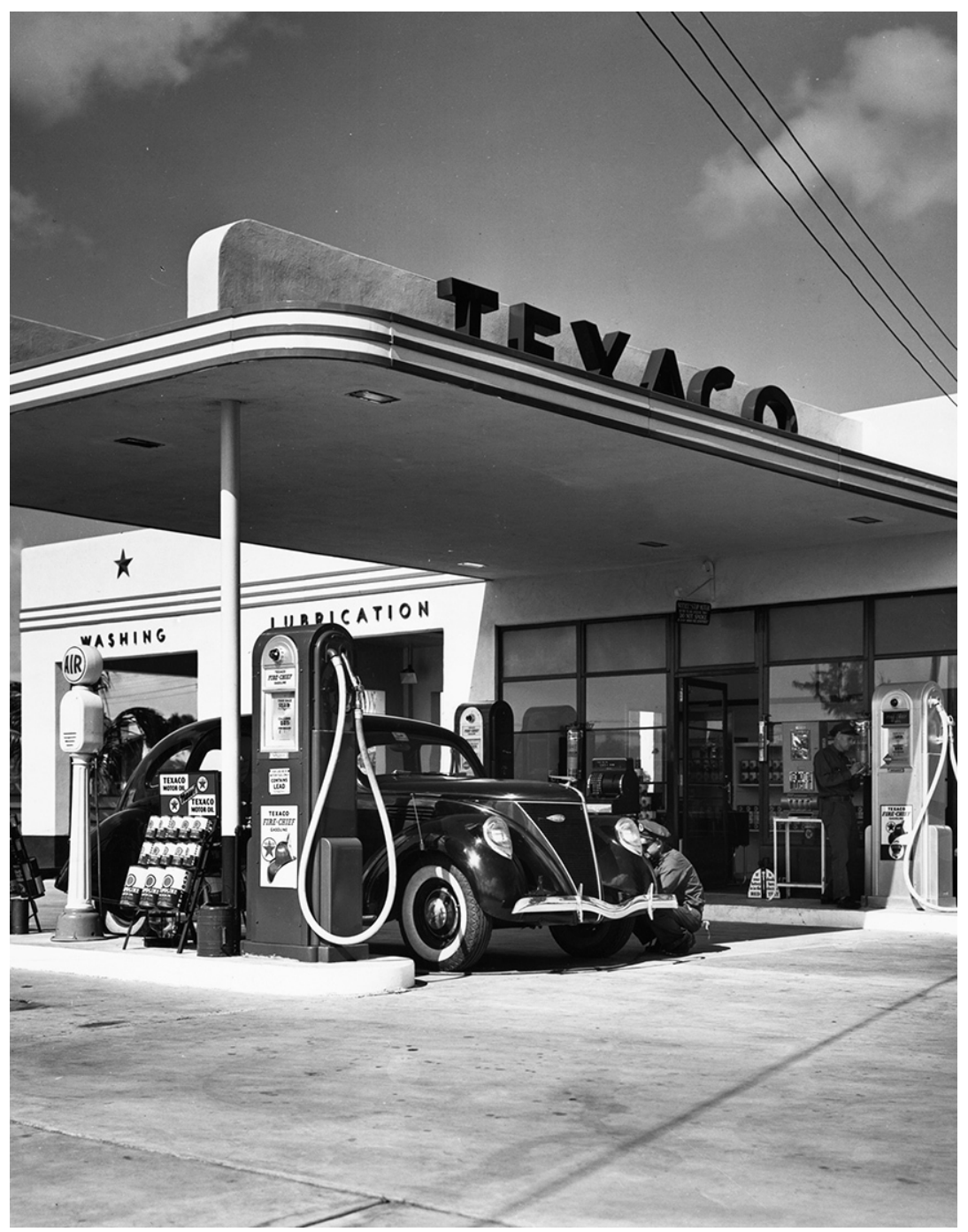

Con el fin de alcanzar este propósito, demandaron los servicios de Walter Dorwin Teague, quien les proporcionó el diseño de una serie de estaciones de servicio de diversa magnitud dependiendo de los servicios prestados, manteniendo una misma identidad: diseños limpios, esquinas curvas redondeadas y detalles en color rojo y verde (Cotter, 2009): edificios styling revestidos con porcelana esmaltada blanca, el mismo color del fondo del logotipo que también rediseñó. Sus diseños se convirtieron rápidamente en un estándar envidiable para otras compañías y en una referencia dentro del mundo del marketing (Silvestre \& Camarasa, 2017).

La compañía petrolífera tejana le encargó sus primeros proyectos para gasolineras en 1934, los cuales se prolongaron en el tiempo durante los siguientes años. Ante los problemas acaecidos con la crisis de 1929, el consumo de combustible había descendido considerablemente, de manera que tanto ésta como otras compañías petrolíferas se vieron en la obligación de dar un giro en su estrategia comercial. Texaco fue de las primeras en apostar por una renovación de sus estaciones de servicio, adaptando las formas al estilo streamline característico de los automóviles (Kwoka, 1993). 
Pero tal vez el mayor avance y el que contribuyó de una forma más notable a la consolidación de su identidad corporativa fue el de la industrialización de las gasolineras. El uso de materiales prefabricados y la facilidad tanto para el montaje como para la construcción - lo cual llevó a Teague a plantear que se podría extrapolar este sistema a la construcción de viviendas y revolucionar el sector (Pulos, 1988) -, permitieron que en menos de una década Texaco construyese en torno a 500 estaciones de servicio repartidas por toda la geografía norteamericana, convirtiéndose en un icono de su tiempo.

Dicho crecimiento aumentó exponencialmente conforme fueron pasando los años, y tras dos décadas más de 20.000 de estas estaciones de servicio, de estilo americano y coronadas por una gran estrella roja, se repartían por todo el mundo. Su prolífico trabajo para Texaco fue, desde el punto de vista arquitectónico, la aportación más relevante de Teague. Sus diseños para las estaciones de servicio de esta corporación significaron un cambio de visión, una ruptura con lo existente, y consiguieron traspasar los objetivos publicitarios de la marca para acabar convirtiéndose en edificios-icono de una era, que se extendían por todo el territorio norteamericano siendo fácilmente reconocibles por los ciudadanos y erigiéndose como un excelente ejemplo de éxito de su identidad corporativa.

El segundo grupo es el de los pabellones diseñados para Ford. En 1934 se llevó a cabo el primer proyecto de pabellón para esta compañía. Éste se ubicaba en Chicago y constaba de una torre de planta circular que comunicaba con otro cuerpo, de mayores dimensiones, que, por su geometría, recordaba a un circo romano. Se trataba de una sala de exposiciones de forma alargada con zonas ajardinadas en el centro. Los itinerarios en el pabellón no estaban predeterminados, por lo que se podía visitar la muestra con total libertad.

El siguiente pabellón encargado por Ford se edificó en San Diego, presentando un diseño innovador que mejoraba la distribución del anterior y en el que, a través de una torre nuevamente de planta circular, se accedía a un edificio también circular con un gran patio en su espacio central donde se encontraban sus vehículos expuestos (Reid, 1937). La intención de Teague se percibe claramente cuando se examina la planta y se aprecia el interés por componer un recorrido que obligase al visitante a recorrer todo el espacio expositivo. Tras revisar el pabellón de Chicago, decidió reorganizar los recorridos, obligando al visitante a realizar un itinerario en el que se mostraban de manera ordenada los automóviles de la compañía. Además, buscando mejorar la experiencia de quienes acudiesen, Teague proyectó una rampa en espiral en la que Ford patrocinó «experiencias de conducción» presentadas como Road of Tomorrow. El edificio fue dotado de una apariencia con influencias del Art Déco, aunque de formas más depuradas.

Entre 1936 y 1937 Ford volvió a recurrir a Teague para que desarrollara los diseños de otro edificio en Dallas con motivo de la Centennial Exhibition. Ahora ya sí de clara estética Art Déco, era un pabellón de colosales dimensiones, pero el presupuesto del que se disponía condicionó la forma. Ésta, en vez de ser curvilínea como en los anteriores pabellones, se proyectó en base a un rectángulo. Teague, en su intervención en el espacio interior, llegó a proponer a Edsel Ford un planteamiento de iluminación en el que los espacios de recepción estuviesen poco iluminados, en contraste con los espacios expositivos, para que éstos y sus dioramas pareciesen «brillantemente iluminados" por comparación (Doordan, 2000).
Su prolífico trabajo para Texaco fue, desde el punto de vista arquitectónico, la aportación más relevante de Teague. 
En esa misma exposición ejecutó un edificio encargado por la compañía Texaco. Éste contaba con unos espacios interiores en los que predominaban las esquinas curvas y los recorridos estaban claramente definidos ${ }^{4}$, buscando la comodidad para los visitantes y una mayor flexibilidad para interactuar con lo expuesto.

Su labor en el campo del diseño arquitectónico le condujo a que la revista American Architect and Architecture le hiciese una entrevista en la que hablaba acerca de los problemas de circulación de los visitantes de las muestras internacionales y los planteamientos que proponía para resolverlos (Teague, 1937).

El tercer grupo es el de los edificios para la Feria Mundial de Nueva York. Cuando se decidió celebrar este evento, se eligió a Teague como uno de los diseñadores que se encargarían de algunos de los proyectos (Marchand, 1991), formando parte además de la junta de diseño de la exhibición. Sus modelos para los pabellones de las compañías Ford y US Steel fueron un éxito, si bien no fue la única exposición para la que proyectó pabellones expositivos con gran afluencia de visitantes.

Uno de los edificios más destacados construido con motivo de la Feria de Nueva York de 1939 fue el pabellón de la US Steel, en el que Teague colaboró con el diseñador G. F. Harrell y los arquitectos York \& Sawyer (Monaghan, 1939). Se trata de un edificio en forma de cúpula de acero inoxidable de color azul. Al caer la noche se iluminaba con luces también azules. El interior quedaba estructurado en torno a dos niveles a través de los que se generaba un recorrido donde el visitante podía conocer la historia de la compañía y el proceso de fabricación del acero.

Otro de los pabellones diseñados por Walter Dorwin Teague con motivo de la exposición fue el proyectado para la compañía DuPont, el cual contaba con una estructura metálica en forma de torre y 32 metros de altura construida con el fin de simular burbujas generadas por productos químicos al ser iluminada por la noche. La estructura estaba unida a otra estructura metálica semicircular que actuaba a modo de escenario. El proyecto lo llevó a cabo conjuntamente con R. J. Harper y el ingeniero A. M. Erickson.

Figura 4.

Pabellón diseñado por Teague para Ford con motivo de la Dallas Centennial Exhibition, 19361937. Fuente: Colección Alfaro Hofmann.

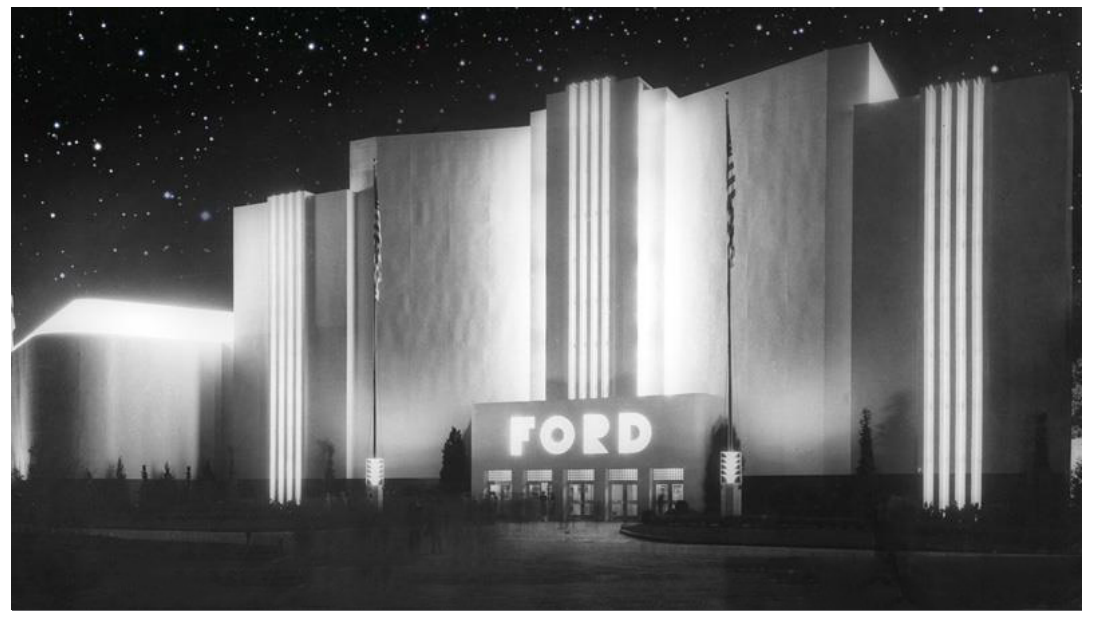

4. El propio Teague se enorgullecería de este proyecto afirmando que: "The Texaco Building at the Texas Centennial in Dallas is an excellent example of planning for dramatic display and controlled traffic flow». (Teague, 1937). 


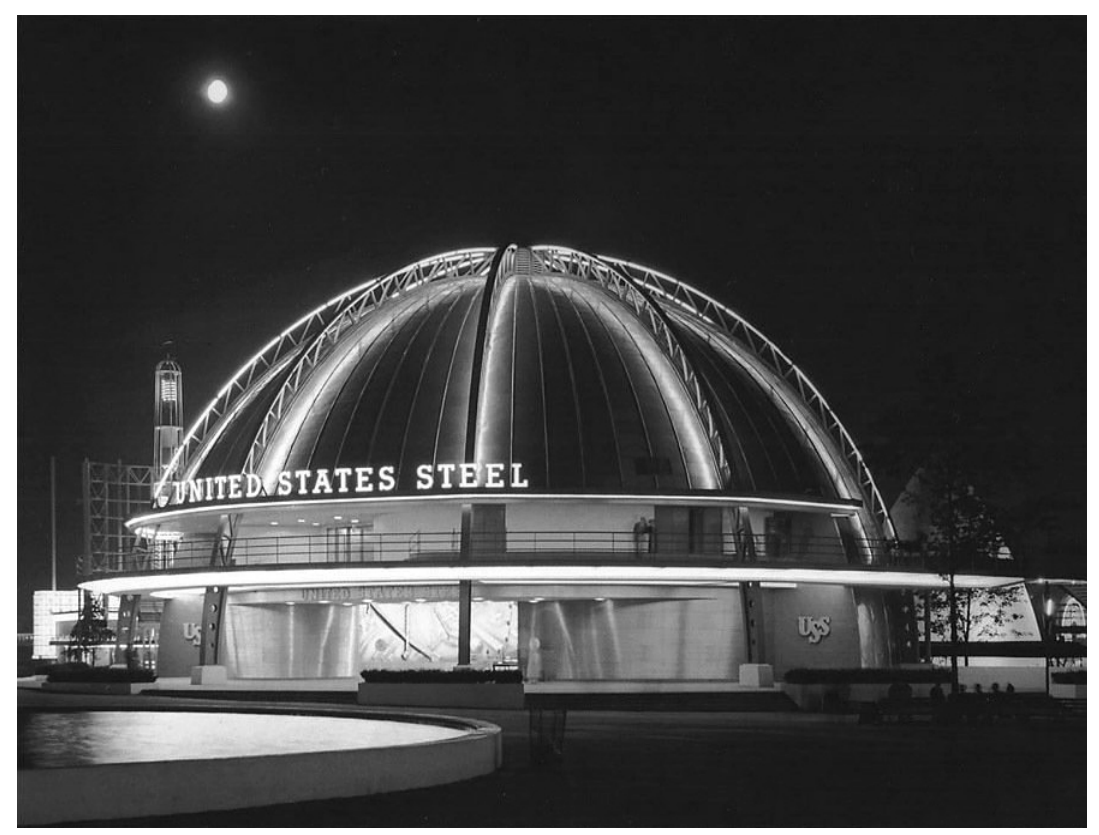

De entre todos los edificios proyectados para aquella exhibición internacional de 1939, el más relevante fue el diseñado por la compañía Eastman Kodak. Se llevó a cabo en colaboración con el diseñador Stowe Myers y el arquitecto Eugène Gerbereux, quienes en equipo proyectaron un edificio que se estructuraba en torno a una estancia de planta semicircular y grandes dimensiones desde la que se accedía a otras dependencias que mantenían el mismo radio de curvatura en sus paredes. De cubierta plana y con grandes ventanales que permitían el paso de la luz al interior, contaba con una planta baja, donde, además del hall de entrada, se encontraba una primera sala de exposiciones, y un segundo nivel, en el que se ubicaba la sala de proyecciones, el restaurante, otra sala de exposiciones y oficinas.

Existían semejanzas en la distribución de los espacios entre éste y el pabellón diseñado para la misma compañía años atrás en San Diego, en el que su intención fue la de crear unas circulaciones que discurriesen de manera perimetral. En el exterior se distinguían dos volúmenes: un primer volumen con el hall, y un segundo cuerpo donde se situaban el resto de dependencias a excepción de la sala de proyección. Una torre exenta de más de veinte metros de altura, de estructura metálica, estaba cubierta por grandes fotografías en blanco y negro de 2,5 por 3,3 metros.

Otros proyectos destacados en el campo de la arquitectura fueron el de la renovación de la sede de la AIGA (American Institute of Graphic Arts) en 1952, institución de la que también fue presidente, y el encargo para la construcción del Centennial Dome en Richmond, Virginia, un proyecto que llevó a término su equipo en 1961 (Silvestre, 2016).

\section{El legado}

Teague falleció en 1960. Desde entonces, su compañía ha seguido en activo, ahora bajo el nombre de Teague, produciendo diseños para grandes firmas, siendo su labor reconocida recientemente con el prestigioso premio Red Dot Design Award. Su
Figura 5.

Pabellón de US Steel para la feria de Nueva York de 1939. Fuente: archive.curbed.com (Consultado el 29 de marzo de 2021).

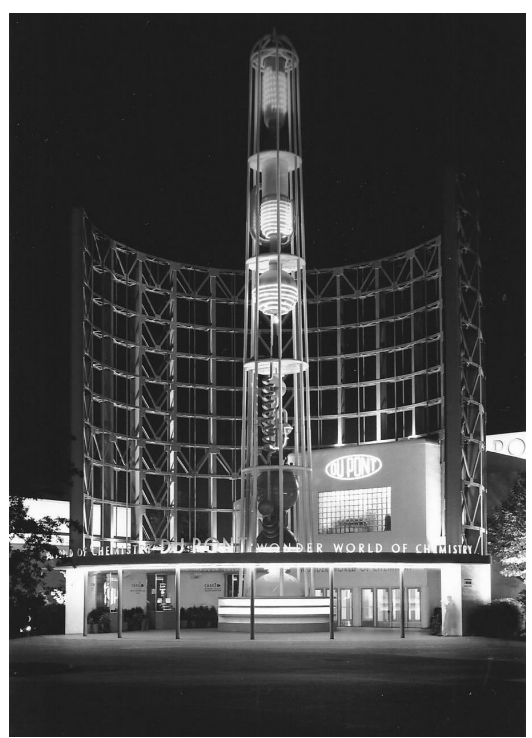

Figura 6.

Pabellón de Dupont en la feria de Nueva York de 1939. Fuente: archive.curbed.com 
Figuras 7 y 8.

Maqueta del pabellón de Kodak diseñado por Walter Dorwin Teague para la Feria de Nueva York de 1939. Fuente: Colección Alfaro Hofmann.

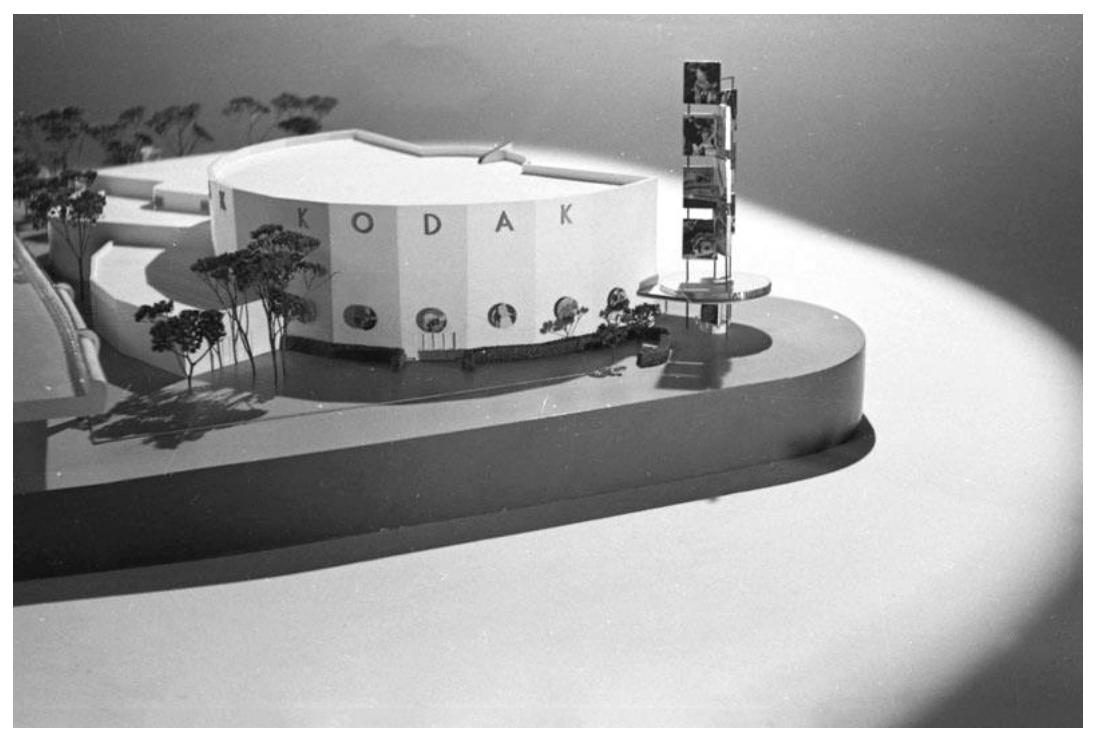

trayectoria ha sido igualmente premiada con numerosos galardones. Después de su muerte, su hijo Walter Dorwin Teague Junior continuó su andadura al mando de la oficina Walter Dorwin Teague Associates. El legado de Teague y su visión perdura hasta día de hoy en su compañía, la cual trabaja con clientes como Hewlett-Packard, Microsoft, Samsung y Boeing, entre otros, y continúa manteniendo una filosofía de trabajo que trasciende la vida de su fundador.

La producción de Walter Dorwin Teague en el campo del diseño es de gran relevancia en la cultura popular del siglo XX, llegando más allá de las fronteras de Estados Unidos con creaciones que acabaron formando parte del día a día de mucha gente. Diseños ergonómicos en los que, como en el caso de otros pioneros estadounidenses (Fairbanks, 1988), trajeron consigo estudios que adaptaban los objetos y las máquinas a la anatomía humana. Jugó un papel capital en la configuración del concepto de identidad corporativa tal y como lo conocemos en la actualidad, llegando más allá que Peter Behrens, pues si bien éste dio forma a la identidad de AEG generando el logotipo, anuncios publicitarios como el de la bombilla incandescente o incluso su fábrica de turbinas, Teague, y atendiendo al caso de Texaco, diseñó, además, un modelo para la producción industrializada de sus propias gasolineras. La expansión de la compañía por toda la geografía estadounidense primero y por otros lugares del planeta después, sirvió para que ese modelo producido en serie se instaurase en lo común, ayudando a una más rápida identificación con la compañía, apoyando a su identidad visual.

Existen actualmente ejemplos como los módulos prefabricados de los restaurantes McDonald's que funcionan de la misma manera. Pero no sólo lo consiguió con Texaco. También hizo lo propio con otras compañías como Kodak, para la que, además de diseñar diferentes modelos de cámaras fotográficas que acabaron convirtiéndose en iconos de la sociedad del momento (Pulos, 1986), también se encargó de proyectar algunos de sus pabellones para sus ferias, o la automovilística Ford, en la que contribuyó al incremento de sus ventas gracias al diseño de unos característicos pabellones con unos identificativos códigos de conducta muy aceptados por el gran público durante las ferias celebradas en la década de los treinta. 


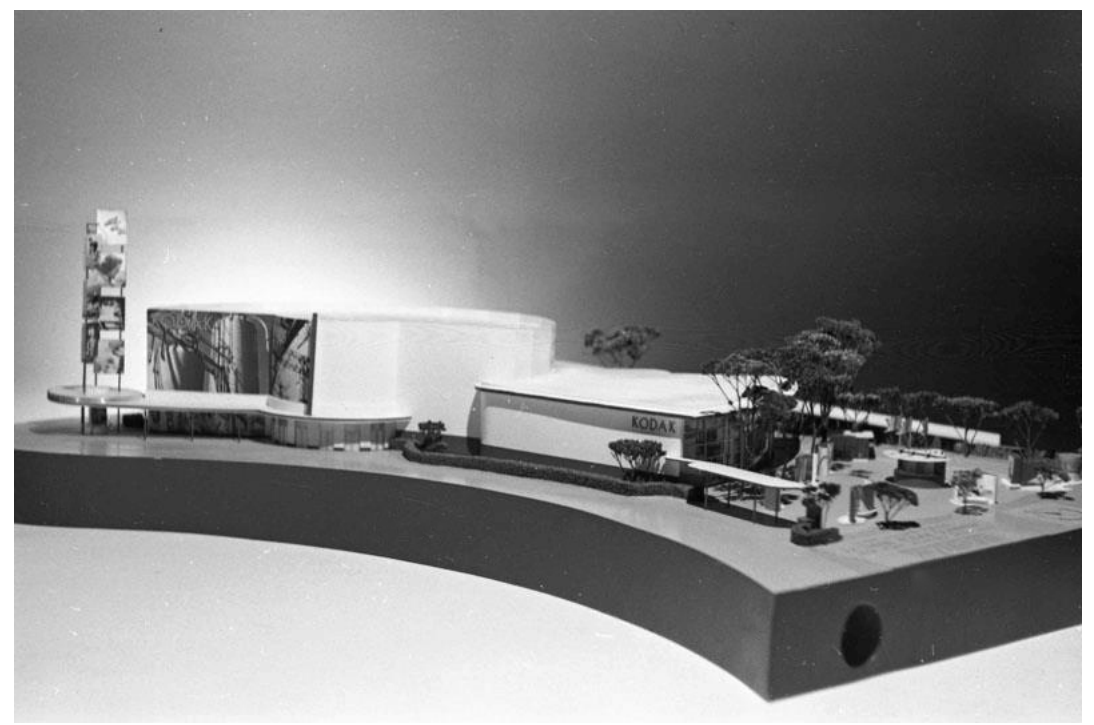

El binomio «diseño asociado a la publicidad» contribuyó también a la potenciación de las identidades visuales de las empresas. En el particular caso del diseñador que centra la atención del presente artículo, en la mayoría de los encargos que recibió, fue más allá de la mera intervención puntual. Se aproximó en gran medida a sus clientes llegando incluso a fidelizar a algunos de ellos. Buscó crear diseños que se convirtiesen en comunes para la sociedad, que contribuyesen a dotar de identidad a la compañía para la que fueron pensados. Con todo, puede considerase a Walter Dorwin Teague como un exponente creador de las primeras identidades corporativas. Un diseñador gráfico que supo adaptarse a las exigencias del mercado de su tiempo y transformarse profesionalmente con éxito para convertirse en uno de los pioneros estadounidenses en el campo del diseño.

Esa adaptabilidad llegó a Europa tiempo después de la mano de figuras tales como Dieter Rams, quien estudió Arquitectura y Diseño Interior y fue contratado por Braun para ejercer como arquitecto. Posteriormente adaptó sus funciones para convertirse en el diseñador más prolífico de la compañía y contribuir a la consolidación y crecimiento de la identidad de marca con sus diez principios del buen diseño.

Y quizá figuras como Phillippe Starck, Piero Lissoni u Oki Sato representan muy bien la transformación profesional en la actualidad, virando de una disciplina a otra, en este caso de la arquitectura al diseño de producto o interior, y trabajando para numerosas compañías internacionales, como Alessi, Vitra o Fritz Hansen. Oki Sato ha tenido como referente al diseñador de moda Issey Miyake, quien, a su vez, se formó en diseño gráfico, transformándose para convertirse en un referente en su sector.

\section{Cuestiones estéticas: Entre el styling y el streamline}

Teague fue uno de los pioneros del diseño estadounidense, contribuyendo a la consolidación del concepto styling. Este adquirió un significado de capital importancia en las décadas centrales del siglo XX, siendo considerado como una estrategia del diseño que tenía por objetivo incrementar el número de ventas y dinamizar el mercado para
Puede considerarse a

Walter Dorwin Teague como un exponente creador de las primeras identidades corporativas. Un diseñador gráfico que supo adaptarse a las exigencias del mercado de su tiempo y transformarse profesionalmente con éxito para convertirse en uno de los pioneros estadounidenses en el campo del diseño. 
En su trabajo para el interior del Boeing 707 llegó a reliar un model a escala real para ensayar de manera rigurosa la adecuación de los asientos y los aspectos ergonómicos del mobiliario mientras en el exterior aplicaba todos los recursos a su alcance para realzar el atractivo de las formas aerodinámicas de la nave. así reactivar el consumo recurriendo al atractivo de sus productos (Rodríguez Ortega, 2002) en un tiempo en el que la superación de la crisis del 29 era la primera y principal necesidad de la población estadounidense. Suponía la continuación de lo mismo bajo ropajes distintos, considerando la actividad del diseñador como un medio para incrementar la facturación de las empresas, quedando subordinada a los intereses del productor.

El objeto concebido de esta manera se escindía en un cuerpo-la máquina propiamente dicha-, y en una envolvente, preparada independientemente de aquel para atraer al comprador en virtud de la apariencia. El «valor estético» se convirtió en el objetivo del diseñador y hoy en día es en el styling, donde radica esa máxima aspiración (Jiménez Julián, 1995). Así, este concepto implicaba un proceso que se iniciaba con el diseño de producto y continuaba hasta su presentación y comercialización.

Pero Teague quiso ir más allá, y al mismo tiempo que defendía las ventajas de la estética styling de los productos y la aplicación sobre los mismos del streamline, entendió la necesidad de una aproximación científico-técnica del diseño (Arceneaux, 1975). En su trabajo para el interior del Boeing 707 llegó a realizar un modelo a escala real para ensayar de manera rigurosa la adecuación de los asientos y los aspectos ergonómicos del mobiliario mientras en el exterior aplicaba todos los recursos a su alcance para realzar el atractivo de las formas aerodinámicas de la nave. De este modo, en muchas de sus creaciones, la eficiencia de la forma jugaba un papel destacado.

Por otro lado, sus diseños se caracterizaron por cierta contención y seriedad, desde los modelos de cámaras para Kodak hasta la imagen corporativa de las gasolineras Texaco, así como las estaciones de servicio que perdurarían sin cambios hasta bien entrados los años 80 y que se convertirían en un icono del siglo XX norteamericano y en un precedente de los nuevos tipos arquitectónicos. Ese carácter icónico contribuiría a la gestación de una identidad propia para cada empresa.

Asimismo, la contención estética de la que se habla permitió dar forma a encargos que acabaron instalándose en el imaginario colectivo, prolongándose su existencia durante años en muchos casos y entendidos por algunas empresas como una parte muy importante para su desarrollo.

\section{Referencias}

Arceneaux, M. (1975). Streamline: art and design of the forties. Ann Arbor: Troubador Press.

Cotter, B. (2009). The 1939-1940 New York World's Fair. Creation and Legacy. San Francisco: Arcadia.

Doordan, D. P. (2000). Design history: An anthology. Cambridge: Massachussets Institute of Technology.

Fairbanks, J. L. (1988). America's measure of mankind: proportions and harmonics. Smithsonian Studies in American Art, 2-1, 73-87.

Feinberg, A. R. (1972). The Kodak collector. Winnetka, Ill.: Alan R. Feinberg.

Gantz, C. (2014). Founders of American Industrial Design. Jefferson, NC: McFarland and Company Inc. 
Gautrand, J. C. (1983). Publicités Kodak: 1910-1939. Paris: Contrejour.

Hall, D., \& Hall, S. G. (2006). American Icons: An encyclopedia of the people, places and things that have shaped our culture. Westport, CT: Greenwood.

Giménez Julián, Emilio (1995) «La cultura industrial. Origen y desarrollo de los aparatos domésticos electrificados», en La mecanització de la casa: Una història de l'electrodomèstic. Valencia: Generalitat Valenciana, pp. 34-49.

Hornung, C. P. (1991). The advertising designs of Walter Dorwin Teague. New York: Art Direction Book.

Jones, W. D. (2003). A field guide to gas stations in Texas. Austin: Texas Department of Transportation.

Kwoka J. E. (1993). The sales and competitive effects of styling and advertising practices in the U.S. auto industry. The Review of Economics and Statistics, 75-4, 649-656.

Lippincott, J. G. (1945). Industrial design as a profession. College Art Journal, 4-3, 149-152.

Marchand, R. (1991). The designers go to the Fair: Walter Dorwin Teague and the professionalization of corporate industrial exhibits, 1933-1940. Design Issues, 8-1, 4-17.

Marquette, C. L. (1952). Flour for man's bread: a history of milling by John Storck and Walter Dorwin Teague. Minneapolis: University of Minnesota Press.

Monaghan, F. (1939). Official Guide Book: New York World's Fair, 1939. New York: Exposition Publications, Inc.

Porter, G. (2002). Raymond Loewy: Designs for a consumer culture. Wilminton: Hagley Museum and Library.

Pulos, A. J. (1986). American design ethic: A history of industrial design of 1940. Boston: MIT Press

Pulos, A. J. (1988). The american design adventure, 1940-1975. Boston: Massachusetts Institute of Technology.

Reid, K. (1937). Walter Dorwin Teague, Master of Design. Design Pencil Points, 9 , pp. $539-574$

Rodríguez Ortega, Nuria (2002). Manual de teoría y estética del diseño industrial. Málaga: Universidad de Málaga.

Silvestre, F. (2016). Pioneros del diseño: Transformación y adaptabilidad de los diseñadores estadounidenses. Buenos Aires: Diseño Editorial.

Silvestre, F., Camarasa, P. (2017). Publicidad y mercado. Estrategias del diseño en la sociedad de la comunicación. I+Diseño, vol. 12, pp. 99-112.

Soloway, R., London, R. (2007). Camera designs of Walter Dorwin Teague. Journal of the photographic historical society of Canada, 32.

Soloway, R., London, R. (2006). Some camera designs of Arthur Crapsey, Henry Dreyfuss and Raymond Loewy. Photographica Digest, 13-11, 8.

Teague, W. D. (1940). Design this day: the technique of order in the machine age. New York: Harcourt, Brace and Co. 
INVESTIGACIÓN / Francisco Silvestre Navarro / Pablo Camarasa Balaguer

Teague, W. D. (1937). Exhibition Technique. American Architect and Architecture, 9. Teague, W. D. (1947). Land of plent: a summary of possibilities. New York: Harcourt, Brace and Co. 